\title{
A Minimum Number of Features with Full-Accuracy Iris Recognition
}

\author{
Ibrahim E. Ziedan \\ Dept. of computers and systems \\ Faculty of Engineering \\ Zagazig University \\ Zagazig, Egypt
}

\author{
Mira Magdy Sobhi \\ Dept. of computers and systems \\ Faculty of Engineering \\ Zagazig University \\ Zagazig, Egypt
}

\begin{abstract}
A minimum number of features for $100 \%$ iris recognition accuracy is developed in this paper. Such number is based on dividing the unwrapped iris into vertical and horizontal segments for a single iris and only vertical segments for dual-iris recognition. In both cases a simple technique that regards the mean of a segment as a feature is adopted. Algorithms and flowcharts to find the minimum of Euclidean Distance (ED) between a test iris and a matching database (DB) one are discussed. A threshold is selected to discriminate between a genuine acceptance (recognition) and a false acceptance of an imposter. The minimum number of features is found to be 47 for single iris and 52 for dual iris recognition. Comparison with recently-published techniques shows the superiority of the proposed technique regarding accuracy and recognition speed. Results were obtained using the phoenix database (UPOL).
\end{abstract}

Keywords-Iris recognition; Iris features; Speed of Iris recognition; Features reduction

\section{INTRODUCTION}

The purpose of 'Iris Recognition', a biometrical-based technology for personal identification and verification, is to recognize a person from his/her iris prints. In fact, iris patterns are characterized by high level of stability, distinctiveness and noninvasive nature. Each individual has a unique iris (as shown in Fig.1); and the difference even exists between identical twins and between the left and right eye of the same subject [3].

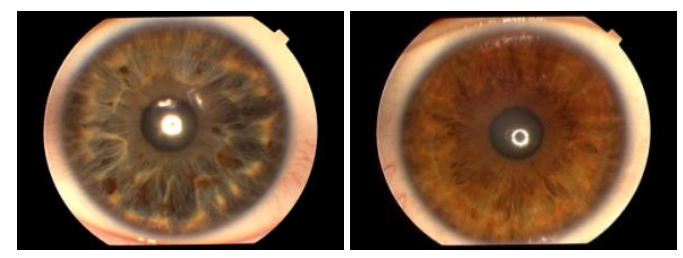

Fig. 1. Distinctiveness of human iris

Recently, iris recognition is becoming one of the most important biometrics used in recognition over fingerprints and facial recognition [2]. Facial recognition is relatively easy to fool. Age, facial hair, surgery, head coverings, and masks all may affect results. Fingerprints are not as accurate as iris recognition as they require physical contact with a scanner device that needs to be kept clean (hygiene issue) [7].
In view of the above, Iris recognition has attracted the attention of many researchers. Conventional techniques like Discrete Cosine Transform (DCT) and Haar Wavelet and a newly introduced one; column-means method were presented in detail in a previous publication by the authors [1].where accuracies of $98.44 \%$ and $97.66 \%$ were achieved.A preceding work by Aly I. Desoky et al. [4] used a technique based on template fusion of several iris images and achieved nearly $99 \%$ accuracy. However the paper is organized as follows:Section II is a general consideration one. Single Iris Recognition is considered in section III. Section IV discusses dual-Iris recognition.Performance results are introduced in section $\mathrm{V}$. Section VI discusses the reduction of feature vector. Section VII presents a comparison between different techniques and paper conclusion is given in section VIII.

\section{GENERAL CONSIDERATIONS}

Features are extracted with different feature extraction methods to encode the unique pattern of the iris into a biometric template. The template that is generated in the feature encoding process may also need a corresponding matching metric, which gives a measure of similarity between two iris templates. This metric should give one range of values when comparing templates generated from the same subject eye, and another range of values when comparing templates created from different subject irises. These two cases should give distinct and separate values, so that a decision can be made with high confidence as to whether two templates are from the same subject iris, or from two different subjects.

The Euclidean Distance (ED) is employed for classification of iris templates [6]. Two templates are considered to be matching if the Euclidean Distance is lower than a specific threshold. As a result, a decision can be made in the matching step, based on threshold values. That is to say that a similarity between two iris images may be evaluated using the ED as compared to a threshold.

The proposed and employed methods may be described through two algorithmic steps. In the first step, an iris template for each image in the database is created and stored as shown in Fig.2. In the second step, an iris template for a query image is created and then a comparison based on ED is made. The system can accept or reject a subject according to the minimum value of ED, as shown in the flow chart of figure (3). 


\section{Step 1: Creating DB Templates}

i. Transform the normalized area of the iris [5] into a rectangular block (unwrapped iris) of fixed dimensions and then normalize (normalization size $=64 \times 512$ )

ii. Compute Column-Means / Combined Rows \& ColumnMeans for each image and store such Means coefficients in vectors with size n (512/576). This is the Features Vector (FV) of that image.

iii. Repeat items $\mathrm{i}$ and ii for every database image.

Step 2: Template of the Query Image

i. A feature vector for the query image may be formed in a similar way to that carried out in Step 1

ii. For a query image ' $q$ ' compute the Euclidean Distance (ED) to every database image 'p', using (1):

$$
\mathrm{ED}=\sqrt{\sum_{\mathrm{i}=1}^{\mathrm{n}}\left(\mathrm{Vp}_{\mathrm{i}}-\mathrm{Vq}_{\mathrm{i}}\right)^{2}}
$$

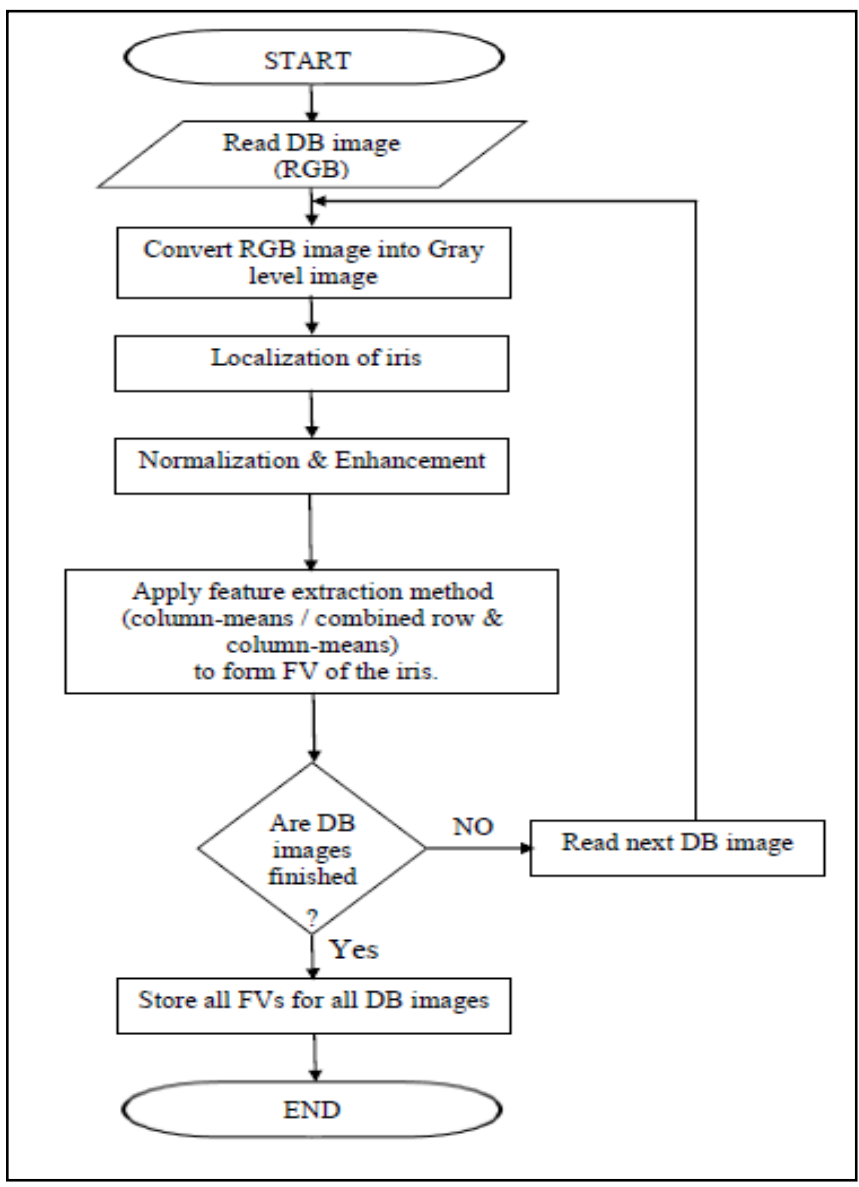

Fig. 2. Flow-chart of DB iris template creation

iii. Determine the DB image with minimum ED that is less than a threshold value. This corresponds to a matching

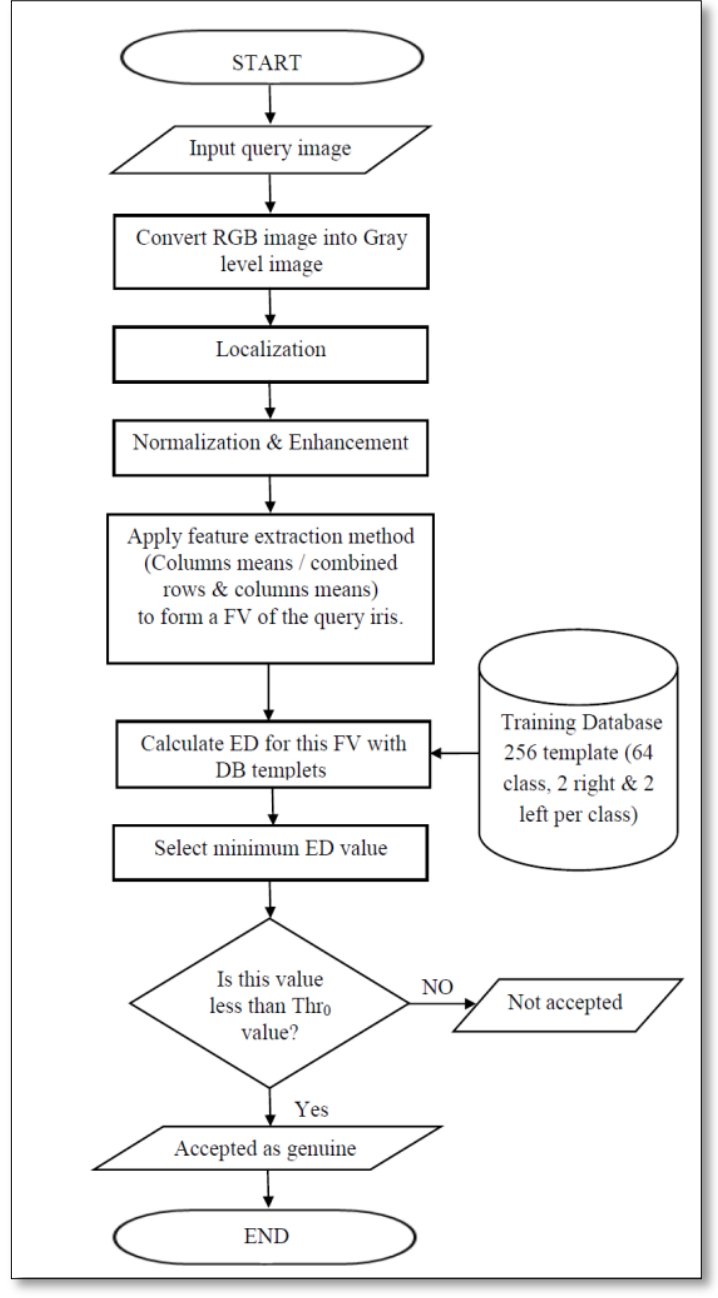

Fig. 3. Flow-chart of a subject identification

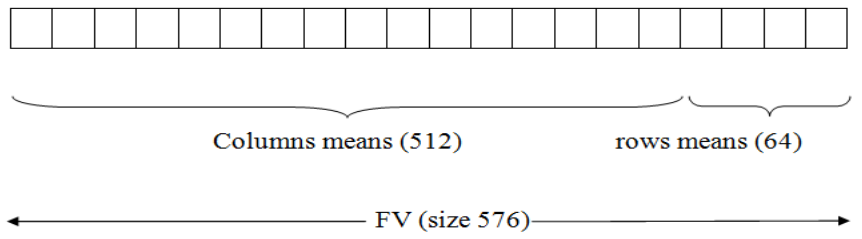

Fig. 4. FV of an eye template using combined rows \& column-means method

between the two images $\mathrm{p}$ and $\mathrm{q}$ as shown in Fig. 5 .

For the combined Rows \& Column-Means method a FV of $n=576$ elements for each image may be formed as shown in Fig. (4).

During performance testing, a test image was considered and compared with all images in the database. The percentage of correct detections (genuine acceptances) is the percentage of relevant images returned and the percentage of incorrect detections is the amount of irrelevant images returned. 
A threshold value must be chosen to determine the best accuracy and optimum (smallest), False Acceptances (FA) and False Rejections (FJ). As stated-above if the Euclidean Distance between two templates is less than the threshold value the templates would have been generated from the same iris and a match occurs (GA). On the other hand if the ED is greater than the threshold value the two templates are considered to have been generated from different irises.

It should be noted that smaller threshold values relate to higher rejections (i.e. less GA) of images belonging to DB subjects(i.e. FJ) while higher threshold values may cause higher false acceptances (FA) of impostor images

\section{SINGLE IRIS RECOGNITION}

In a single iris recognition both the Column-Means method and the Rows and Column-Means method are adopted as given in section II. The definition of accuracy as employed in this study may be expressed as;

Where:

$$
\text { Accuracy }=\frac{\mathrm{GA}}{\mathrm{GA}+\mathrm{FA}+\mathrm{FJ}}
$$

GA represents the no. of genuine acceptances,

FA represents the total no. of false acceptances and

FJ is the no. of false rejections.

This study is based on using phoenix DB where testing of 128 images with a no. of $256 \mathrm{DB}$ ones was performed.

N.B. It should be noted that a query image of one subject iris may be regarded as an image of an imposter when excluding other images of the same subject from the DB set. Therefore the no. of false acceptances obtained may be considered as applied to both irises belonging to the DB and those of subjects from outside the DB (imposters) as well.

The identification accuracy obtained with combined rows \& column-means method at different threshold values is shown in Fig. 5, where a maximum of $99.22 \%$ accuracy was achieved at a threshold value of 3.7 .

Fig. 6 shows an example for the ED of a test image of index 1(right eye) to the $256 \mathrm{DB}$ images with three threshold values $(3,5$, and 3.7). One threshold value is small (3) and exhibits a false rejection of the test image. The second value is relatively high (5) and shows many false acceptances. This situation may be considered as resulting from imposter's irises as indicated above. The third value which is the optimum (3.7) shows a true matching between the test image and the two images of the same eye belonging to the same subject.

\section{DUAL IRIS RECOGNITION}

Iris recognition using both eyes of an individual has not been extensively investigated. However, for an iris recognition at a distance, capturing a good quality image of the same eye at different times is a challenging task and so a dual-iris approach is potentially beneficial.

For each subject, there are 4 images in the database; 2 left eye images and 2 right eye ones. Calling these images L1,

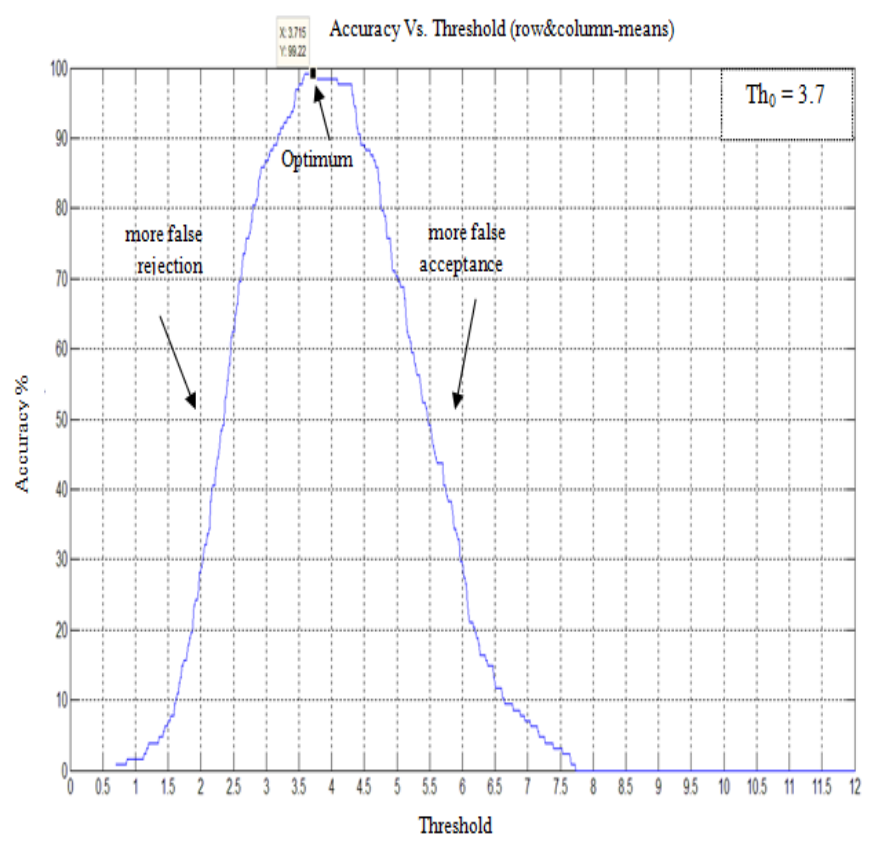

Fig. 5. Identification accuracy obtained for Combined Row \& ColumnMeans Method with Threshold

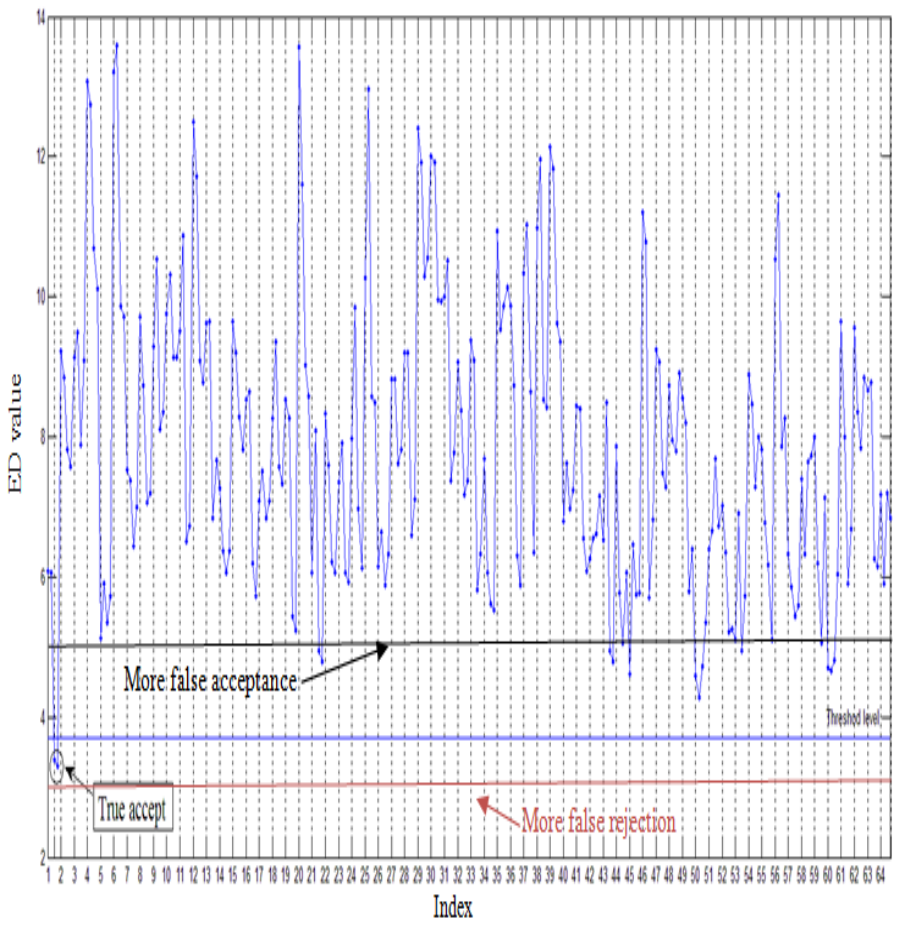

Fig. 6. Recognition of a test iris of index1with 256 DB images using Combined Rows \& Column-Means Method

L2, R1 and R2, then four new templates may be formed for each subject denoted by L1R1, L1R2, L2R1 and L2R2. Hence 


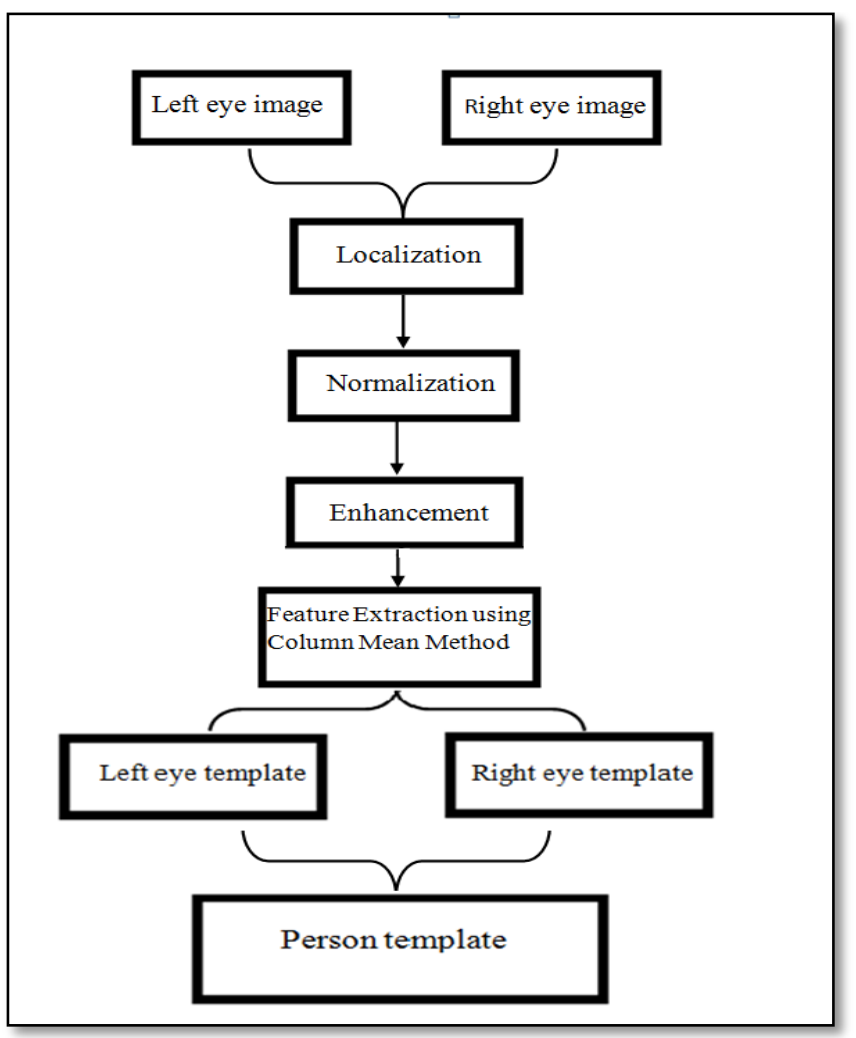

Fig. 7. Steps required for forming a subject's templates for dual iris recognition

test images contain one left eye and one right eye image that forms one template for each subject. Steps required for forming a subject's templates are shown in Fig. (7).

The mean of each column in the normalized image is

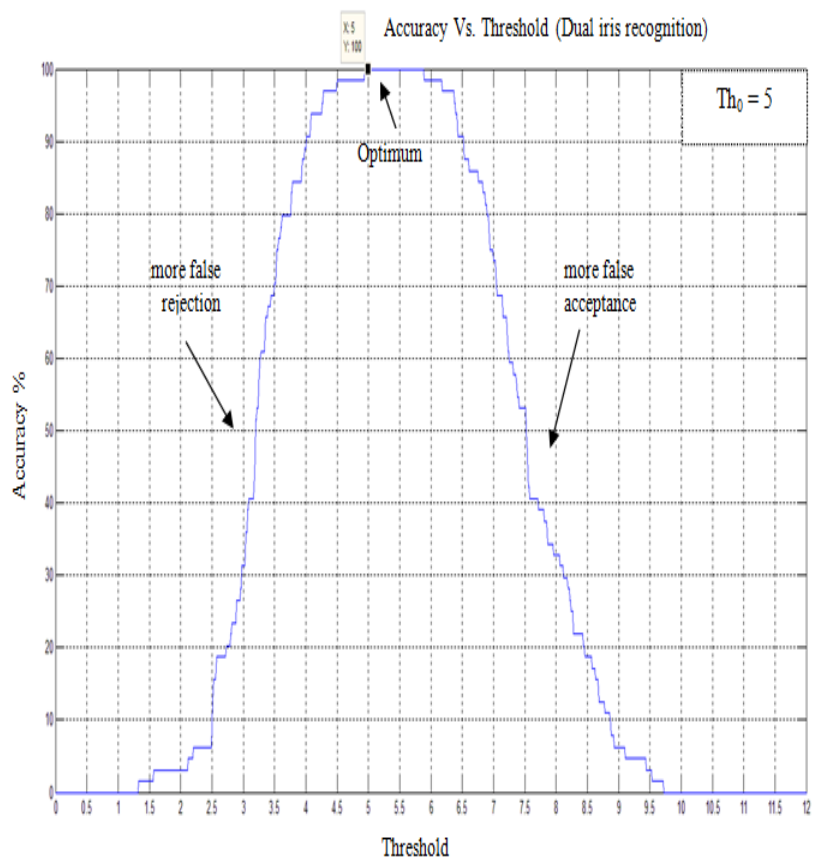

Fig. 8. Identification accuracy obtained for Dual -Iris recognition using Column-Means Method computed for the two eyes. The resulting column-means form the FV of dual-iris images with a size of 1024 elements. Fig.8 shows the accuracy obtained with dual-iris recognition using column-means method at differentt threshold values.A $100 \%$ accuracy is shown at a threshold of 5 .

\section{PERFORMANCE RESUlts}

A comparison between the recognition rate (accuracy) as obtained in the previously-published results [1] using Haar wavelet and DCT techniques and the proposed one are presented in Table I that shows also the corresponding optimum thresholds..

A comparison between Feature-Vector lengths for each method is presented in Table II.

As shown in Table I the feature-vector length of the HaarWavelet transform method is the smallest one, but the method gives the lowest accuracy.

\section{REDUCTION OF FEATURE -VECTOR LENGTH}

\section{A. Column-means method}

To reduce storage requirements and improve execution speed the FV length ought to be reduced.

TABLE I. The Percentage of ReCognition Rates

\begin{tabular}{|l|l|l|}
\hline matching Techniques & $\begin{array}{l}\text { Recognition } \\
\text { rate }\end{array}$ & Threshold \\
\hline Haar Wavelet Transform & $97.66 \%$ & 4.9 \\
\hline DCT(Discrete Cosine Transform) & $98.44 \%$ & 4.4 \\
\hline Column-Means Method & $98.44 \%$ & 3.7 \\
\hline $\begin{array}{l}\text { Combined Rows \& Column-Means Method } \\
\text { Dual iris recognition using Column-Means } \\
\text { method }\end{array}$ & $\mathbf{1 0 0 \%}$ & $\mathbf{5}$ \\
\hline
\end{tabular}

TABLE II. FEATURES- Vector LENGTHS FOR DIFFERENT METHODS

\begin{tabular}{|l|l|}
\hline Techniques & F.V. length \\
\hline Haar wavelet transform & $\mathbf{4 8 0}$ \\
\hline DCT & 3072 \\
\hline Column-means method & 512 \\
\hline Combined row\& column-means method & 576 \\
\hline Dual iris recognition using column-means method & 1024 \\
\hline
\end{tabular}




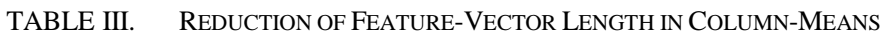
METHOD

\begin{tabular}{|l|l|l|l|}
\hline $\begin{array}{l}\text { No. of } \\
\text { columns }\end{array}$ & $\begin{array}{l}\text { FV } \\
\text { length }\end{array}$ & No. of segments & $\begin{array}{l}\text { Accuracy (Column- } \\
\text { Means Method) }\end{array}$ \\
\hline 1 & 512 & $512[1$ col.] & $98.44 \%$ \\
\hline 2 & 256 & $256[2$ col.] & $98.44 \%$ \\
\hline 4 & 128 & $128[4$ col.] & $98.44 \%$ \\
\hline 8 & 64 & $64[8$ col.] & $98.44 \%$ \\
\hline 9 & 57 & $56[9$ col. $]+1[8$ col. $]$ & $98.44 \%$ \\
\hline 10 & 51 & $50[10$ col. $]+1[12$ col. $]$ & $98.44 \%$ \\
\hline 11 & 47 & $46[11$ col. $]+1[6$ col. $]$ & $98.44 \%$ \\
\hline 12 & 43 & $42[12$ col.] $+1[8$ col.] & $98.44 \%$ \\
\hline 13 & 40 & $39[13$ col.] $+1[5$ col.] & $97.66 \%$ \\
\hline 14 & 37 & $36[14$ col.] $+1[8$ col.] & $96 \%$ \\
\hline 16 & 32 & $32[16$ col.] & $95.31 \%$ \\
\hline
\end{tabular}

The unwrapped iris is divided into vertical segments each comprising a number of columns. Tests were performed where the number of columns per segment is changed from 1 to 16 . A feature may be specified in terms of the mean per segment instead of a column mean. An optimum (maximum) number of columns per segment may be reached while maintaining the highest accuracy of recognition which is $98.44 \%$ (Table I). Results are presented in Table III where the optimum number of columns per segment is 12 . This means that the FV length is reduced to only 43 features.

\section{B. Combined horizontal and vertical segments}

The same method can be applied to decrease the feature vector length of a combined rows \& column-means method. Dividing the unwrapped iris height (64 rows) into horizontal segments starting with one row per segment was done. Combining $\mathrm{ED}_{1}$ of such horizontal segments with $\mathrm{ED}_{2}$ of the vertical ones taking different weights $\mathrm{w}_{1}$ and $\mathrm{w}_{2}$ where $\mathrm{w}_{1}+\mathrm{w}_{2}=1$, the ED of two templates used for such method was calculated by using the formula:

$\mathrm{ED}=\mathrm{w}_{1} \times \mathrm{ED}_{1}$ (Row-segment means) $+\mathrm{w}_{2} \times \mathrm{ED}_{2}$ (Column-segment means)

Weighting factors were changed so as to reach the best recognition rate with the minimum no. of segments vertically and horizontally (maximum no. of columns or rows per segment).

The results presented in Table IV show that the optimum values of weights $\mathrm{w}_{1}$ and $\mathrm{w}_{2}$ are 0.3 and 0.7 for the horizontal and vertical segments, respectively. This result is based on maintaining the accuracy at $100 \%$ while changing the number
TABLE IV. ACCURACY OF COMBINED HORIZONTAL \& VERTICALSEgMENT MEANS METHOD AT DifFERENT WEIGHTS

\begin{tabular}{|c|c|c|c|c|c|c|}
\hline \multirow[b]{2}{*}{$\begin{array}{l}\text { No. of } \\
\text { Col. }\end{array}$} & \multirow[b]{2}{*}{$\begin{array}{l}\text { No. of } \\
\text { rows }\end{array}$} & \multicolumn{5}{|c|}{ Accuracy } \\
\hline & & $\begin{array}{l}w_{1}=0.1 \\
\& \\
w_{2}=0.9\end{array}$ & $\begin{array}{l}w_{1}=0.2 \\
\& \\
w_{2}=0.8\end{array}$ & $\begin{array}{l}w_{1}=0.3 \\
\& \\
w_{2}=0.7\end{array}$ & $\begin{array}{l}w_{1}=0.4 \\
\& \\
w_{2}=0.6\end{array}$ & $\begin{array}{l}w_{1}=0.5 \\
\& \\
w_{2}=0.5\end{array}$ \\
\hline 1 & 1 & 99.22 & 99.22 & 99.22 & 99.22 & 99.22 \\
\hline 2 & 1 & 99.22 & 99.22 & 99.22 & 99.22 & 98.44 \\
\hline 4 & 1 & 100 & 99.22 & 99.22 & 99.22 & 98.44 \\
\hline 8 & 1 & 100 & 100 & 99.22 & 99.22 & 96.09 \\
\hline 12 & 1 & 100 & 100 & 99.22 & 97.66 & 94.5 \\
\hline 12 & 2 & 99.22 & 100 & 99.22 & 99.22 & 96.88 \\
\hline 12 & 4 & 99.22 & 100 & 100 & 99.22 & 99.22 \\
\hline 12 & 8 & 99.22 & 99.22 & 100 & 100 & 99.22 \\
\hline 12 & 16 & 98.44 & 99.22 & 100 & 100 & 100 \\
\hline
\end{tabular}

of columns and rows per segment. The minimum number of features was achieved with an optimum number of 12 columns per a vertical segment and 16 rows per a horizontal segment as shown in Table IV.

Accordingly the feature-vector length can be reduced using the principle of horizontal and vertical segments to 47 only instead of 576; as indicated in Table V.

\section{Dual iris recognition based on vertical segments}

The same method of vertical-segments division can be applied to dual-iris recognition based on column-means method. Originally the method has a feature vector length of.

TABLE V. FEATURe Vector LENGTHS IN COMBined Rows \& COLUMNSegments Means Method $\left(\mathrm{W}_{1}=0.3, \mathrm{~W}_{2}=0.7\right)$

\begin{tabular}{|l|l|l|l|}
\hline $\begin{array}{l}\text { No. of } \\
\text { columns/ } \\
\text { segment }\end{array}$ & $\begin{array}{l}\text { No. of } \\
\text { rows/ } \\
\text { segment }\end{array}$ & Feature vector length & Accuracy \\
\hline 1 & 1 & $576(512+64)$ & 99.22 \\
\hline 2 & 1 & $320(256+64)$ & 99.22 \\
\hline 4 & 1 & $192(128+64)$ & 99.22 \\
\hline 8 & 1 & $110(64+46)$ & 99.22 \\
\hline 12 & 1 & $107(43+64)$ & 99.22 \\
\hline 12 & 2 & $75(43+32)$ & 99.22 \\
\hline 12 & 4 & $59(43+16)$ & 100 \\
\hline 12 & 8 & $52(43+8)$ & 100 \\
\hline 12 & 16 & $47(43+4)$ & 100 \\
\hline 12 & 32 & $45(43+2)$ & 97.66 \\
\hline
\end{tabular}


1024 elements (512 for each eye). This feature vector length can be reduced using a segment-division technique as employed above. Tests were carried out while changing the number of columns per segment for both irises. An optimum (maximum) number of columns per segment were reached while keeping the accuracy of recognition at $100 \%$. This number was found to be 20 columns per segment reducing the FV length to only 52 instead of 1024; as obvious in table (6).

\section{COMPARISON BETWEEN DIFFERENT TECHNIQUES}

Comparison between different techniques regarding accuracy, no. of features and speed of recognition is presented in table (7).

It is obvious from table (7) that $100 \%$ accuracy may be achieved with one iris using combined horizontal \& vertical segments method in only $5.6 \mathrm{msec}$. for feature extraction and recognition of a test image. This is the best recognition method using one iris. These results are based on utilizing a machine with dual-core processor and a frequency of $2.7 \mathrm{GHz}$

TABLE VI. FEATURE-Vector LENGTH IN DUAL IRIS ReCogNition BASED ON SEGMENT-MEANS METHOD

\begin{tabular}{|l|l|l|l|}
\hline No. of columns & FV length & No. of segments & Accuracy \\
\hline 1 & 1024 & $2 *(512[1$ col. $])$ & $100 \%$ \\
\hline 2 & 512 & $2 *(256[2$ col. $])$ & $100 \%$ \\
\hline 4 & 256 & $2 *(128[4$ col. $])$ & $100 \%$ \\
\hline 8 & 128 & $2 *(64[8$ col. $])$ & $100 \%$ \\
\hline 16 & 64 & $2 *(32[16$ col. $])$ & $100 \%$ \\
\hline 17 & 62 & $2 *(30[17$ col. $]+1[2$ col. $])$ & $100 \%$ \\
\hline 18 & 58 & $2 *(28[18$ col. $]+1[8$ col. $])$ & $100 \%$ \\
\hline 19 & 54 & $2 *(26[19$ col. $]+1[18$ col. $])$ & $100 \%$ \\
\hline 20 & 52 & $2 *(25[20$ col. $]+1[12$ col. $])$ & $100 \%$ \\
\hline 21 & 50 & $2 *(24[21$ col. $]+1[8$ col. $])$ & $98.44 \%$ \\
\hline
\end{tabular}

TABLE VII. Final Comparison BetweEn the PRoposed Methods and OTHERS

\begin{tabular}{|l|l|l|l|}
\hline Techniques & $\begin{array}{l}\text { Recognition } \\
\text { rate }\end{array}$ & $\begin{array}{l}\text { FV } \\
\text { length }\end{array}$ & $\begin{array}{l}\text { Execution time of } \\
\text { FV extraction\& } \\
\text { matching (msec.) }\end{array}$ \\
\hline Wavelet & $97.66 \%$ & 480 & 20 \\
\hline DCT & $98.44 \%$ & 3072 & 135 \\
\hline Vertical segments Method & $98.44 \%$ & 43 & 5 \\
\hline $\begin{array}{l}\text { Combined vertical \& } \\
\text { horizontal segments } \\
\text { Method }\end{array}$ & $100 \%$ & 47 & 5.6 \\
\hline $\begin{array}{l}\text { Dual iris Recognition based } \\
\text { on vertical segments }\end{array}$ & $100 \%$ & 52 & 9.5 \\
\hline
\end{tabular}

\section{CONCLUSIONS}

This paper introduced three different methods to enhance the performance of iris recognition system. The contribution aspects of this work included the enhancement of the iris recognition accuracy, the enhancement of the system's speed during feature-vector extraction stage and recognition stage. The enhancement is mainly a result of the reduction of featurevector length. The first proposed method is the vertical segments-based features with accuracy of $98.44 \%$. The second method is a combined vertical \& horizontal segments-based feature with accuracy of $100 \%$. A dual-iris recognition system based on vertical segments only gave a $100 \%$ recognition rate as well.

A smaller size of feature vector contributes to speeding up the matching stage. However, an optimal feature -vector length of 52 elements for dual-iris recognition and 47 elements for one iris with combined horizontal \& vertical segment-means recognition was reached. Both achieved accuracy (recognition rate) of $100 \%$.

Comparisons between the introduced approaches as regards accuracy, feature extraction and matching time and featurevector length were presented in detail.

The recognition time of Dual-Iris method is approximately $75 \%$ more than that of the combined horizontal \& verticalsegments method of one iris. Their values are 9.5 and $5.6 \mathrm{msec}$. respectively. Matching time only for both methods was found to be closer to each other (1.5and $1.1 \mathrm{msec}$.) than feature extraction time (8 and $4.5 \mathrm{msec}$.).

\section{REFERENCES}

[1] Ibrahim Ziedan and Mira M. Sobhi, "Comparison between Haar wavelet transform,DCT and proposed column- mean method-based Iris encoders," EIJEST, Vol.17, No.2, 2014.

[2] G. P. Khetri, V. P. Pawar, D. C. Jain "Human Computer Interface Through Biometric Iris Recognition System,"International Journal of Computer Science \& Engineering Technology (IJCSET), Vol. 3, No. 7, July 2012.

[3] Karen Hollingsworth, Kevin W. Bowyer, Stephen Lagree, Samuel P. Fenker, Patrick J. Flynn, "Genetically Identical Irises Have Texture Similarity That Is Not Detected By Iris Biometrics," Computer Vision and Image Understanding, Volume 115, Issue 11, November 2011, Pages 1493-1502.

[4] Aly I. Desoky, Hesham A. Ali, Nahla B. Abdel-Hamid " Enhancing iris recognition system performance using template fusion" 10th IEEE International Symposium on Signal Processing and Information Technology (ISSPIT) Luxor, Egypt, December 15-18, 2010

[5] L. Masek and P. Kovesi. MATLAB Source Code for a Biometric Identification System Based on Iris Patterns. The School of Computer Science and Software Engineering, The University of Western Australia. 2003.

[6] Ya-Ping Huang, Si-Wei Luo, En- Yi Chen, “An efficient iris recognition system", International Conference on Machine Learning and Cybernetics, pp. 450-454, 2002.

[7] "Biometric Comparison Guide", https://epic.org/privacy/surveillance/spotlight/1005/irid_guide.pdf, last access 6/1/2015.

[8] Database: Iris database is available on http://phoenix.inf.upol.cz/iris/download/ 